\title{
Article
}

\section{CATCHA: Real-Time Camera Tracking Method for Augmented Reality Applications in Cultural Heritage Interiors}

\author{
Piotr Siekański ${ }^{1, *(\mathbb{D}}$, Jakub Michoński $^{1}{ }^{\mathbb{D}}$, Eryk Bunsch $^{2}$ and Robert Sitnik ${ }^{1}$ \\ 1 Faculty of Mechatronics, Warsaw University of Technology, ul. św. Andrzeja Boboli 8, 02-525 Warszawa, \\ Poland; j.michonski@mchtr.pw.edu.pl (J.M.); r.sitnik@mchtr.pw.edu.pl (R.S.) \\ 2 Museum of King Jan III's Palace, ul. Stanisława Kostki Potockiego 10/16, 02-958 Warszawa, Poland; \\ jerzywart@yahoo.de \\ * Correspondence: p.siekanski@mchtr.pw.edu.pl
}

Received: 6 November 2018; Accepted: 13 December 2018; Published: 15 December 2018

\begin{abstract}
Camera pose tracking is a fundamental task in Augmented Reality (AR) applications. In this paper, we present CATCHA, a method to achieve camera pose tracking in cultural heritage interiors with rigorous conservatory policies. Our solution is real-time model-based camera tracking according to textured point cloud, regardless of its registration technique. We achieve this solution using orthographic model rendering that allows us to achieve real-time performance, regardless of point cloud density. Our developed algorithm is used to create a novel tool to help both cultural heritage restorers and individual visitors visually compare the actual state of a culture heritage location with its previously scanned state from the same point of view in real time. The provided application can directly achieve a frame rate of over $15 \mathrm{~Hz}$ on VGA frames on a mobile device and over $40 \mathrm{~Hz}$ using remote processing. The performance of our approach is evaluated using a model of the King's Chinese Cabinet (Museum of King Jan III's Palace at Wilanów, Warsaw, Poland) that was scanned in 2009 using the structured light technique and renovated and scanned again in 2015. Additional tests are performed on a model of the $\mathrm{Al}$ Fresco Cabinet in the same museum, scanned using a time-of-flight laser scanner.
\end{abstract}

Keywords: CATCHA; camera tracking; cultural heritage; real-time; point clouds

\section{Introduction}

In Augmented Reality (AR), virtual objects are superimposed on a real-world view observed by a user. The main requirement for an immersive AR application is correct spatial alignment between the real-world objects and virtual ones, which means that the virtual objects must be placed in the same coordinate system as the real objects. If the camera moves, their 2D projections on the screen must move accordingly. To achieve this, a precise camera pose, relative to the world coordinate system, must be known. This process is often called camera pose estimation or camera tracking.

Multiple camera tracking methods are available, such as magnetic, inertial, GPS-based, vision, and other methods [1]. Inasmuch as our interest is in cultural heritage interiors, usually guarded by rigorous conservatory policies, we focus on a vision-based camera tracking algorithm, which does not require physical integration into the interior geometry, as compared with alternative solutions that employ external tracking devices. Moreover, we consider only the visible-light spectrum because the use of infrared light may be dangerous for pigments used to paint the walls [2].

In recent years, 3D scanning has become an increasingly popular technique in cultural heritage documentation. Until now, many cultural heritage sites have been digitized using different techniques 
such as laser scanning [3], Digital Photogrammetry (DP) in which the Structure from Motion (SfM) algorithm is widely used [4], or structured light [2] resulting in precise 3D documentation. These high-quality scans may be used in model-based camera tracking algorithms to create specialized AR applications.

The aim of our work is to develop an AR system called Camera Tracking for Cultural Heritage Applications (CATCHA) with the following functionalities.

1. It should work on any interior model, both planar and non-planar, regardless of its registration technique. The solution should depend only on point cloud data.

2. It should work in a wide range of object distances from 0.5 to $2 \mathrm{~m}$.

3. It should be as simple as possible. Neither input data/parameters nor any configuration and initialization in a known initial position should be required from the user.

4. It should work in real-time, i.e., it must have an interactive frame rate of at least $15 \mathrm{~Hz}$ on a mobile device.

5. It should not use any artificial markers.

6. It should exhibit stable visualization with negligible drift and jitter.

To our knowledge, no system has been developed that satisfies all these requirements. CATCHA was implemented and tested in two real cultural heritage interiors scanned using structured light and laser techniques.

Our paper is organized as follows. Related work is presented in the next section, and Section 3 presents the materials used in the investigation. The details of our method are provided in Section 4. Verification of the algorithm on real data and the results are presented in Section 5 and discussed in Section 6, respectively. The last section contains our conclusions.

\section{Related Work}

Markerless camera tracking approaches can be divided into two main categories [5]: relative (image based) and tracking-by-detection (model based). A summary of these approaches is listed in Table 1 . The first category exploits frame-to-frame spatiotemporal information to determine the camera motion between frames. This approach is very sensitive to occlusions and rapid camera movements, and suffers from drift because of the accumulation of tracking errors. It does not require a model of the tracked object to be known beforehand. On the other hand, the tracking-by-detection approach requires a model and relies on extracted features (points, lines, etc.) from both the model and current camera frames. They are independently matched in every frame, and thus, this method does not suffer from the drift effect and can handle partial occlusions and rapid camera movements at the cost of higher computational complexity.

Table 1. Comparison of the relative and tracking-by-detection approaches.

\begin{tabular}{ccc}
\hline Method & Relative Tracking & Tracking-by-Detection \\
\hline Tracking & Image based & Model based \\
Model of a tracked object & May be constructed on the fly & Must be known in advance \\
Robustness to partial occlusions & Low & High \\
Robustness to rapid camera movement & Low & High \\
Drift & Yes & No \\
Computational complexity & Lower & Higher \\
\hline
\end{tabular}

Combined approaches are also available, such as in [6], that employ relative tracking to track the camera motion between consecutive frames and the tracking-by-detection approach to recover from tracking failures and compensate the drift.

We are also aware of the SLAM (simultaneous localization and mapping) [7] based methods that simultaneously track the camera pose and compute the geometry of the surrounding environment. 
Therefore, it does not depend on a predefined model. However, extracting the real-world scale information from a SLAM-based system may be challenging. Further, displaying a specific object content is not directly possible. Thus, model-based tracking is more suitable.

As mentioned earlier, three main techniques are available in capturing point clouds in cultural heritage applications, but many model-based camera tracking algorithms require the model to be built using one particular method. For example, in the last decade, an enormous progress in SfM-based location recognition was achieved [8,9]. In addition, some approaches are available that estimate the camera pose based on laser scans. Although very accurate, they are not suitable for real-time applications [10,11].

With regard to general point cloud based camera pose estimation algorithms, Wu et al. [12] proposed a method to extract SIFT (Scale Invariant Feature Transform) [13] descriptors out of orthonormalized patches that exploit the 3D normal direction of a single patch. However, their approach relies on fronto-parallel surfaces for an initial match, and features are then extracted relative to this direction. This algorithm was originally designed to interactively compute the camera pose in a single query image. However, large errors may be produced when the camera is close to the object surface, as presented in [14], and thus fail to fulfill Requirement No. 2 presented earlier.

Jaramillo et al. [15] proposed a framework that uses a point cloud constructed by an RGBD sensor and thereafter only uses a monocular camera to estimate the pose. In contrast to the SLAM-based approaches, this solution does not track points from frame to frame. Instead, previous poses are only used to limit the search space. Their solution was reported to work at only approximately $3 \mathrm{~Hz}$. Therefore, Requirement No. 4 is not satisfied.

Crombez et al. [16] used laser scanners to create a dense point cloud model of an interior and then used a fisheye camera and synthetic views to localize its position. However, their method requires a manual initialization step. This solution does not fulfill Requirement No. 3.

Probably, a solution most similar to that presented in this paper is the work from Rambach et al. [17]. They rendered approximately 10000 training images of a model and then chose the best matching set. Subsequently, they used relative tracking combined with the tracking-by-detection approach to recover from tracking failure. In the event of a tracking failure, their initialization procedure takes more than $100 \mathrm{~ms}$, which may be visible as a frame drop; thus, their approach may not satisfy Requirement No. 6 in some circumstances.

With respect to markerless camera tracking algorithms in cultural heritage, many solutions rely on available libraries and track only the planar structures in the scene. To name a few, Vanoni et al. [18] created a visual see-through AR application that maps multimodal data on a renascence fresco in Florence, Italy. Their solution relies on the Vuforia [19] library. Gîrbacia et al. [20] used AR to recreate sculptures in the Black Church in Brasov, Romania. In their project, the Instant Player library [21] was used. Verykokou et al. [22] used specially designed algorithm to track the feature points on a planar wall to recreate an ancient stoa in Athens, Greece. All mentioned solutions track only planar structures; thus, they do not satisfy Requirement No. 1. Battini and Laudi [23] tracked a statue of Leonardo da Vinci in Milan, Italy. In their project, ARmedia SDK [24] was used. Compared with the approaches mentioned earlier, this library allows 3D camera tracking based on object texture and non-planar geometry but requires time-consuming manual data preparation and algorithm parameters tuning; thus, Requirement No. 3 is not satisfied.

\section{Materials}

Our algorithm was used to track the camera pose in the King's Chinese Cabinet in the Museum of King Jan III's Palace at Wilanów, Warsaw, Poland. This interior was scanned using structured light technique in 2009 shortly before renovation, and scanned again in 2015 [2]. Figure 1 shows the visual comparison between these two scans. The point cloud registered in 2009 contains 4 billion points and was scanned using SLR cameras, and that registered in 2015 contains 35 billion points and was scanned using industrial cameras with color calibration [25]. In both scans, every point contains its position, 
color, normal vector, and quality value that represents the certainty of a registered point. Based on these two scans, we designed an AR application that allows a user to see the previous state of the Cabinet. Additional tests were performed on the Al Fresco Cabinet in the same museum. This interior was scanned once using the FARO Focus3D X130 HDR time-of-flight (TOF) laser scanner. The cloud contains 172 million points and has the same internal structure.

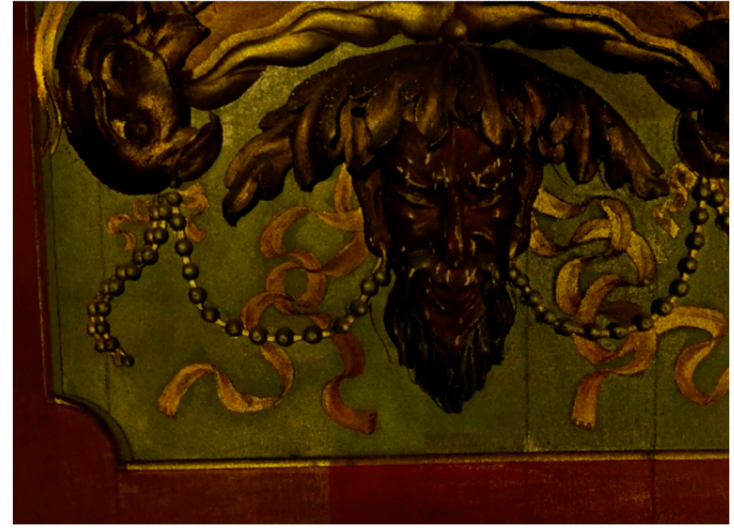

(a)

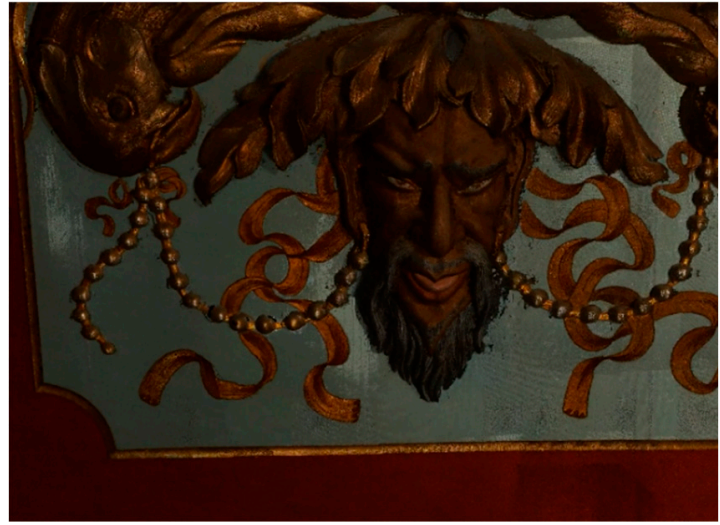

(b)

Figure 1. Visual comparison between two registered point clouds of the King's Chinese Cabinet: (a) from 2009 and (b) from 2015.

In our camera tracking solution, we used a single Logitech B910 camera with the resolution reduced from $1920 \times 1080$ pixels to $640 \times 480$ pixels (Figure 2 shows a sample frame). We connected it to a Lenovo Yoga convertible laptop with Intel i5-5200U 2,20 GHz processor and Nvidia GT 840M graphics card. We implemented our solution using Microsoft Visual Studio 2015 in C++ and OpenCV library on the Windows 8.1 Operating System. We also tested the streaming version of our approach, in which only the image acquisition and final model rendering were performed on the device, and all processing algorithms were remotely executed on a PC with Intel i7-6700K 4.0-GHz processor and Nvidia GeForce 960 (see Section 3 for details). For streaming, we decided to separately send every frame with optional JPEG-LS compression realized using the CharLS library [26]. The application based on CATCHA is shown in Figure 3.

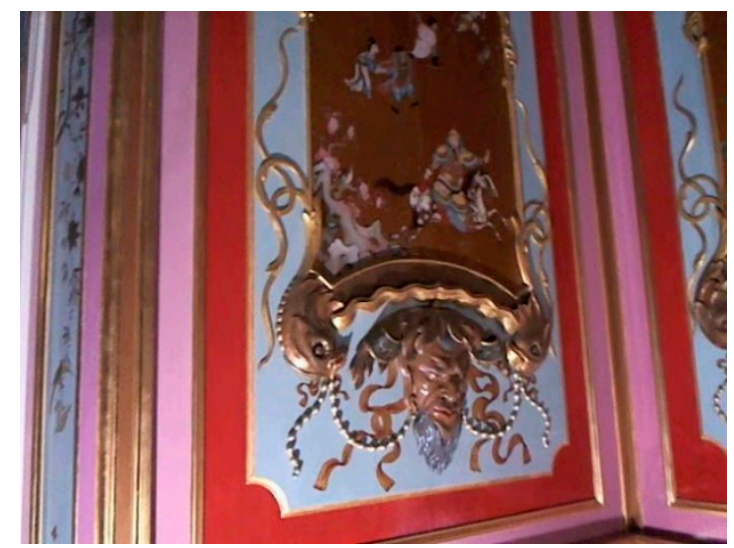

Figure 2. Sample frame taken using a Logitech B910 camera. 


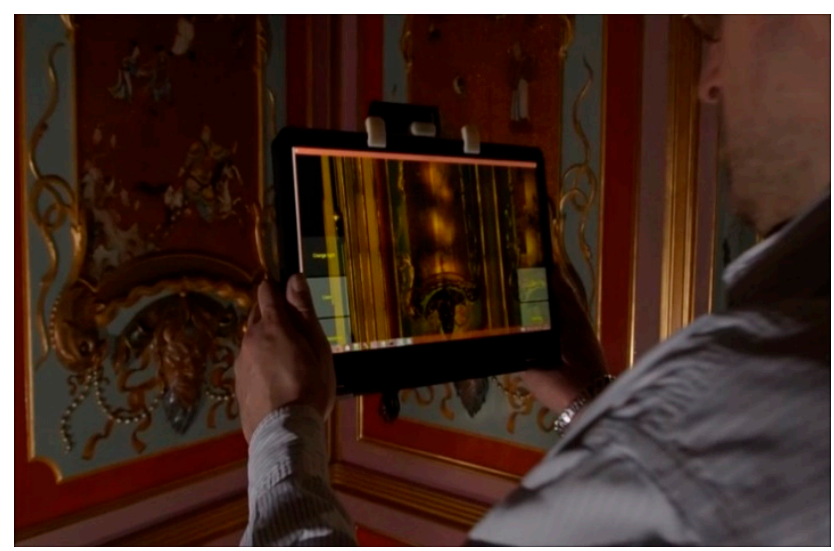

Figure 3. CATCHA-based application in use.

\section{Method}

To track the camera pose relative to the model, we must determine rotation matrix $R$ and translation vector $t$ of the camera in the model coordinate system in each frame. Given camera intrinsic matrix $K$ from the calibration and a set of correspondences between 3D model points $P$ and image points $p$, both in homogeneous coordinates, we must determine the camera extrinsic matrix that minimizes the reprojection error between $p$ and projected model points $p^{\prime}$.

$$
p^{\prime}=K \cdot[R \mid t] \cdot P
$$

Therefore, to determine the camera pose, valid 3D-2D correspondences between the 2D points in the current camera image and the 3D model points must be known.

We assume that CATCHA can work on any point cloud-based data without prior knowledge of their origin. We consider three main sources of the clouds of points: structured light, TOF, and DP. To achieve model origin invariance, we decide to generate high-resolution orthographic renders (Figure 4) with the associated 3D coordinates in every pixel using an approach similar to that in [12].

We segment the point cloud using normal vectors to search for suitable nearly planar surfaces. Then we place the virtual orthographic camera in front of each planar area and render it. In the case of two analyzed interiors, five orthomaps are generated for each interior. If an interior has more complicated geometry, more orthomaps have to be generated. On each render, we extract the keypoints and their descriptors. In our case, the SIFT descriptor extracted by the GPU is used, but we can exploit any descriptor by taking advantage of considerably faster binary descriptors (e.g., ORB [27]) if necessary. Because we store the 3D coordinates of each pixel, we can easily obtain the 3D-2D correspondences between each point found in the orthomap and the associated model point.

This approach leads us to a sparse interior model regardless of the initial point cloud density. In our case, we only extract a few thousand keypoints to cover the entire interior geometry. Further, reduction in the model complexity can be achieved by creating masks on the orthomaps (Figure 5). Mask is a simple binary image with the same size as the orthomap. The black areas on the mask indicate that these areas are rejected from further processing. The purpose of this process is to filter out points and areas of noise and the noise near the borders between the model and its background, as well as the reflective or transparent fragments of a surface. The masks have to be created by the user. We used a semi-automated method based on hue to filter the areas. 


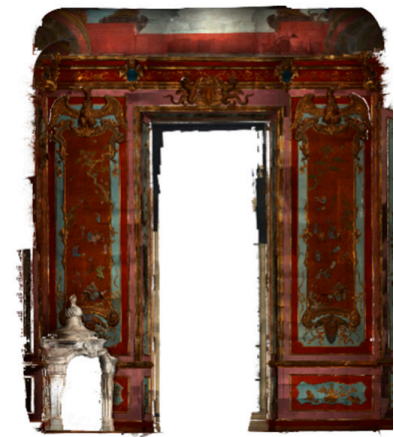

(a)

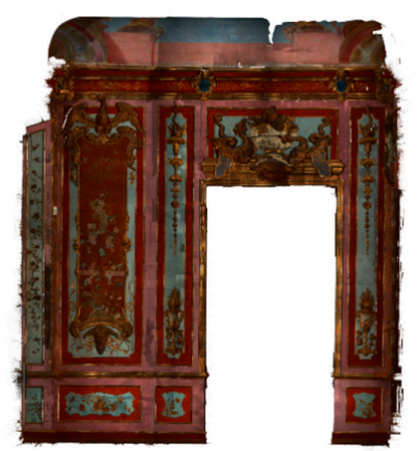

(d)

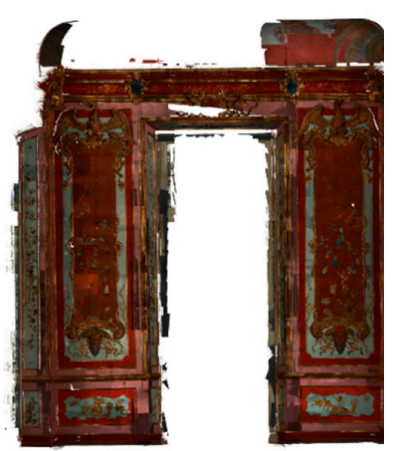

(b)

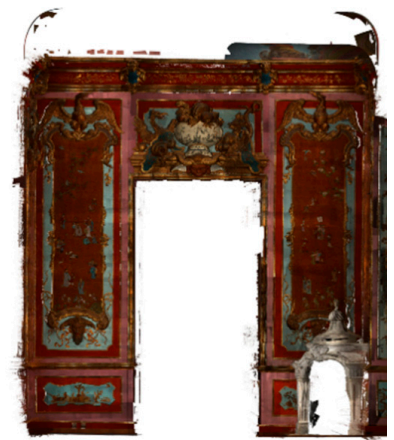

(c)

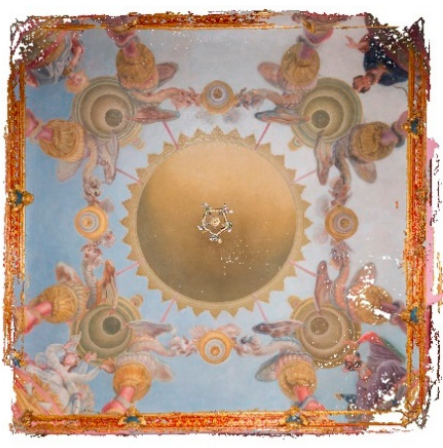

(e)

Figure 4. Generated orthographic renders. (a-d) Four walls of the Cabinet. (e) The ceiling.

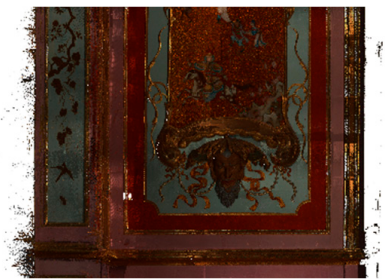

(a)

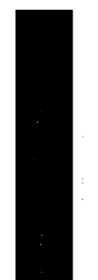

(b)

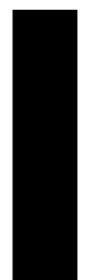

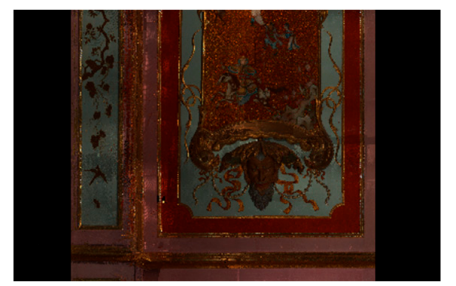

(c)

Figure 5. Illustration of the masks: (a) raw orthomap, (b) mask, and (c) result of filtration.

Depending on the type of interior, a user can be interested in bigger or smaller details. The optimal orthomap resolution must be computed depending on the assumed average distance between the camera and observed wall [we call it later the optimal viewing distance (OVD)]. In our tests, the application smoothly works from approximately 50\% to 200\% of the OVD. In our case, we set this parameter to $1 \mathrm{~m}$. Desired orthomap resolution depends on OVD, camera resolution, its FOV and the real-world size of the interior.

From Figure 6, we obtain:

$$
x=\tan \frac{\varphi}{2} \cdot 2 d
$$

where $\varphi$ indicates the camera field of view (FOV), $d$ is distance to the wall, and $x$ denotes the observed fragment dimension.

We want to maintain the scales of the keypoints as closely as possible. Thus, we intend to keep the ratio between camera resolution $\operatorname{res}_{\mathcal{C}}$ (in pixels) and real-world dimension of observed fragment $x$ (in meters) equal to the ratio between orthomap resolution res $_{O}$ (in pixels) and real-world dimension of the interior $w$ (in meters) (Figure 7).

$$
\frac{x}{r e s_{c}}=\frac{w}{r e s_{o}}
$$


From Equations (2) and (3), we obtain:

$$
r e s_{o}=\frac{w \cdot r e s_{c}}{\tan \frac{\varphi}{2} \cdot 2 d}
$$

Therefore, we propose equation (4) to compute the desired resolution. If we substitute our values, namely, $\varphi=78^{\circ}$, which is the FOV of a Logitech B910 camera, res $s_{c}=640$ pixels, and $w=6 \mathrm{~m}$, we can draw a plot of $d$ against res (Figure 8). In our case, we set OVD to $1 \mathrm{~m}$, which allows the application mentioned in Section 3 to run smoothly from approximately 0.5 to $2 \mathrm{~m}$. Therefore, we decide to render orthomaps with a 2048-px resolution because it has the closest power of two to the chosen OVD (1 m).

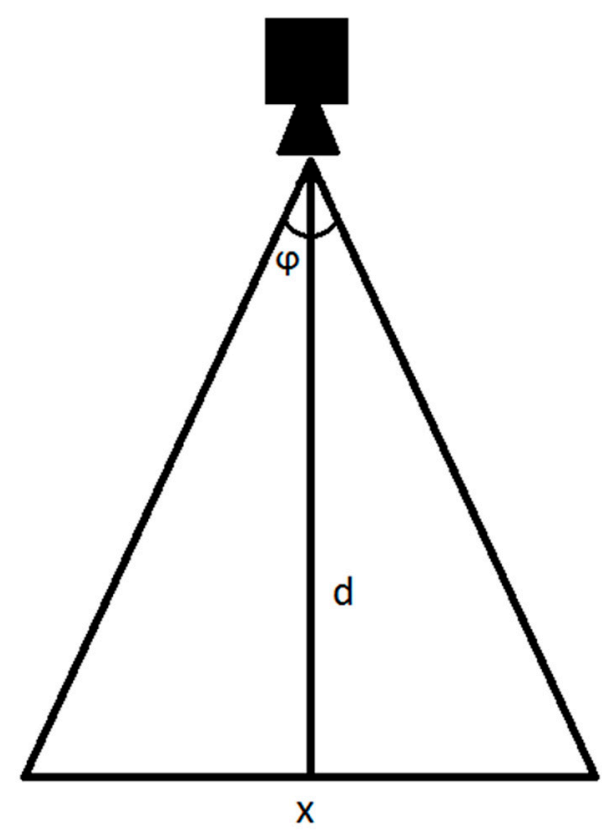

Figure 6. Camera FOV.

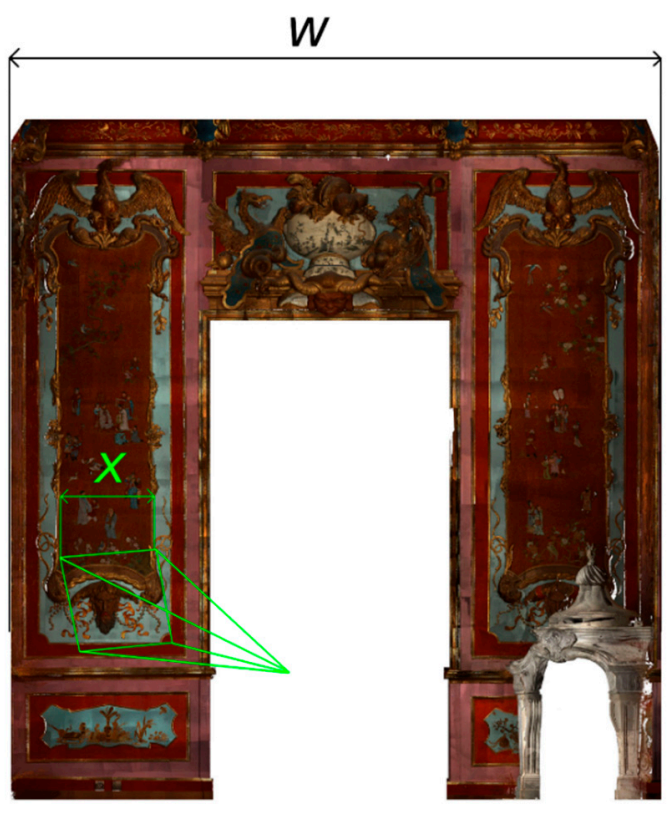

Figure 7. Real-world size of interior $\mathrm{w}$ and the size of observed fragment $\mathrm{x}$. 


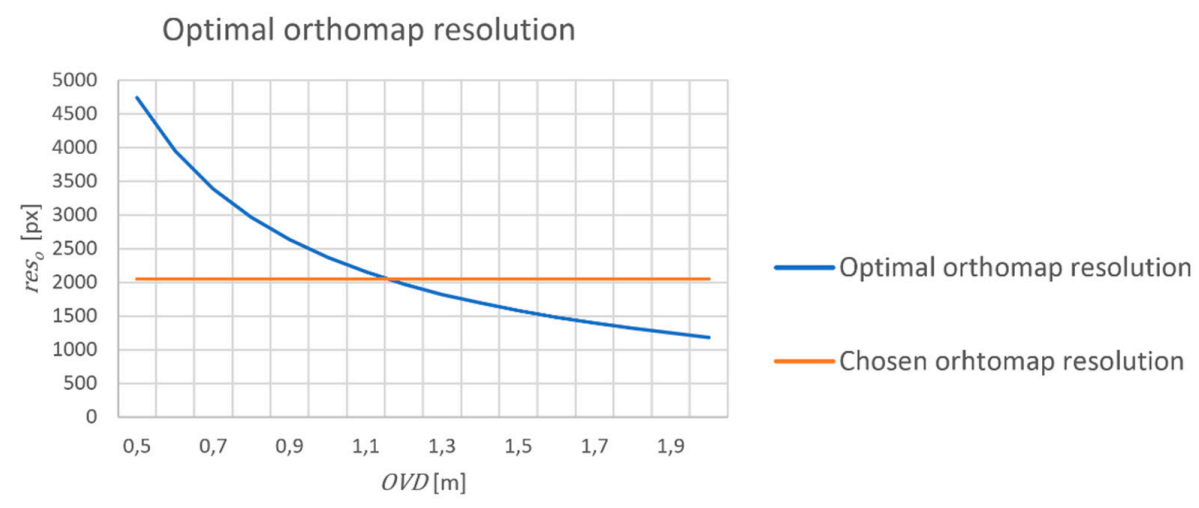

Figure 8. Orthomap resolution $r s_{o}$ and OVD: optimal resolution with respect to optimal viewing distance (blue) and closest power of 2 for viewing distance equal to $1 \mathrm{~m}$ (orange).

The model distinctiveness strongly depends on the quality of the renders. The lesser the noise in the rendered image is, the more reliable and stable are the keypoints. Therefore, we decide to render the orthomaps using $4 x$ antialiasing. We render them with four times bigger resolution and downscale them four times using bicubic interpolation to reduce the noise (Figure 9). A detailed review of the different point cloud rendering methods with focus on descriptor extraction can be found in [28]. As the paper suggests, we do not need splatting because our data is dense enough to render the point cloud without gaps, even with resolution four times higher in each dimension.

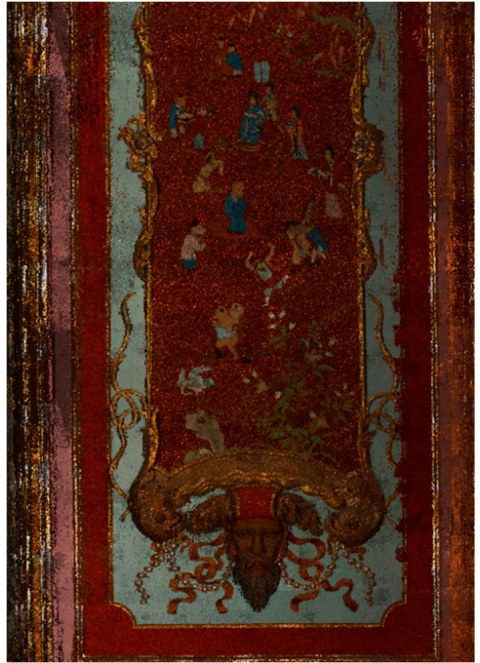

(a)

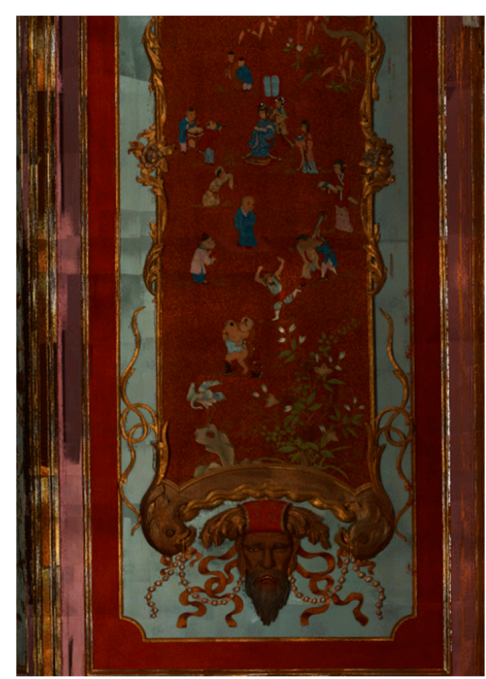

(b)

Figure 9. Comparison between orthomaps rendered in different resolutions: (a) native resolution and (b) with four times of antialiasing.

Our runtime diagram (Figure 10) is similar to the state-of-the-art solutions. For every frame captured by the camera, we extract the keypoints and compute the descriptors. Because our model is very sparse (a few thousand keypoints), we can very effectively match it using a brute-force method on the GPU. We are aware that more methods are available to much more effectively match the descriptors on a CPU [29], but matching on the GPU allows us to avoid costly data transfer between the descriptors extracted on the GPU and matches on the CPU side. Later, the matched keypoints are filtered using the ratio test proposed by Lowe [13]. Then, the camera pose is computed using the EPnP (Efficient Perspective-n-Point) method [30] supported by RANSAC (RANdom SAmple Consensus) sampling in which the P3P algorithm [31] is used. The minimum required number of correspondences is 4, but we reject all the estimations that have less than 7 inliers to ensure that the camera pose is estimated correctly. The camera pose is then transferred to the visualization module. We take advantage of the 
fact that the camera is tracked inside a room; thus, if the computed camera pose is outside it, we reject the pose and do not send it to the visualization module. If the frame is rejected, the previous frame is rendered. We separately compute the camera pose in each frame and later smooth the camera trajectory using Gaussian filtration [32] to reduce jitter in the camera movement.
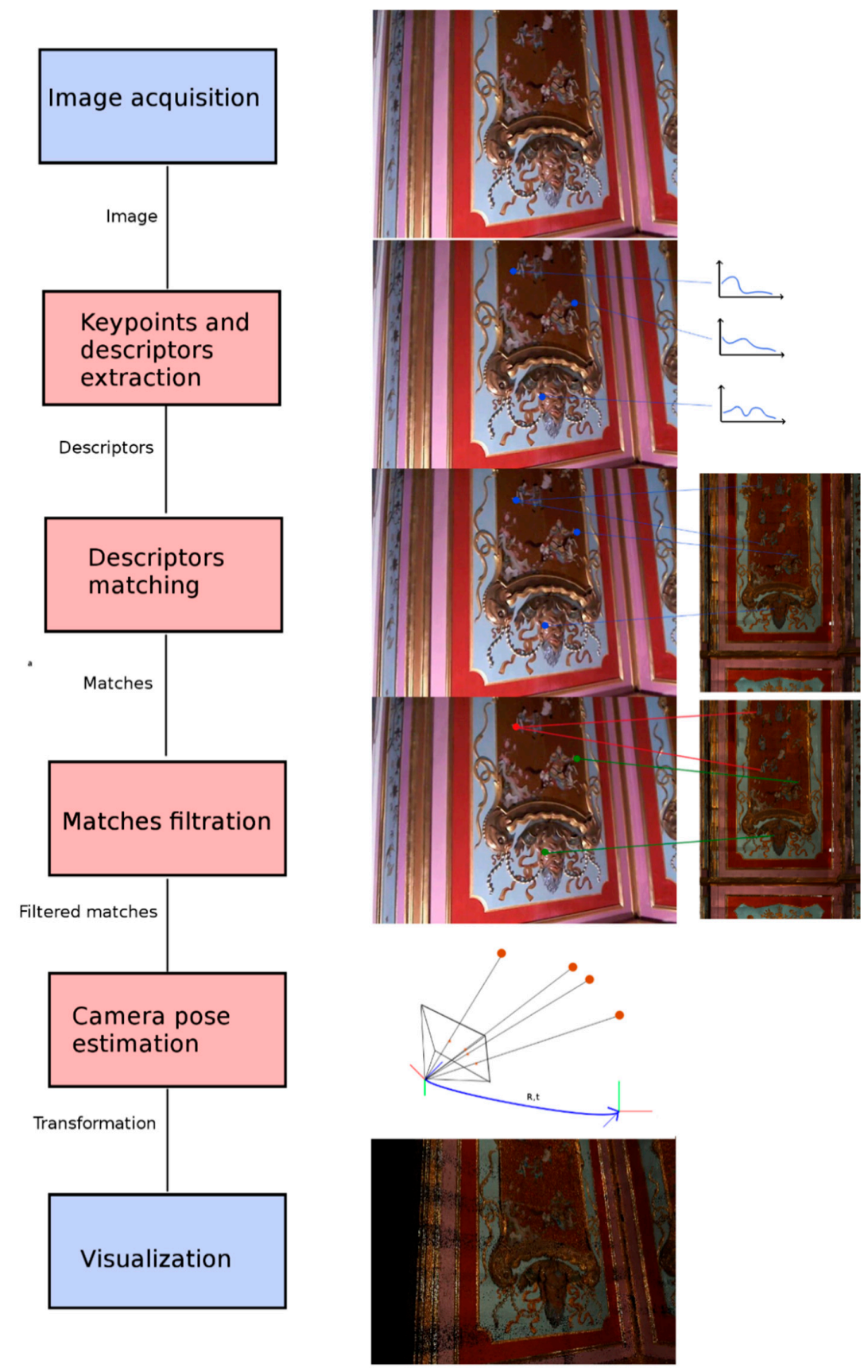

Figure 10. Runtime diagram: steps indicated with violet are performed on the mobile device; those in pink can be remotely computed.

Because our approach relies on orthomaps generated parallel to the walls, processing of the frames with high angular difference from this direction could be challenging. This problem in descriptor matching is called angular invariance. Standard wide-baseline descriptors such as SIFT or SURF (Speeded Up Robust Features) [33] have limited angular invariance of up to 30 [34]. Thus, a very low number of correct matches (if any) are present if the angle between the camera axis and normal to the wall is large. This problem is clearly apparent when the ceiling of the room is very high and the user is 
looking at the elements located just under the ceiling (Figure 11). To overcome this type of problems, the ASIFT (Affine SIFT) [35] algorithm may be used, but the computational complexity is much too high for a real-time application. We differently deal with this problem by dividing the interiors into two main categories based on the ceiling altitude.

1. The average altitude in which the details located just under the ceiling do not require extreme viewing angle and camera tracking is based on the walls.

2. High ceiling in which the problem described above may occur. To overcome this problem, we exploit the fact that the ceiling may also have a rich texture (for instance, it may be covered by frescoes). We rotate the physical camera to point up to the ceiling so that it is always nearly parallel to the camera sensor plane. We estimate the camera position based on the ceiling and rotate it by $90^{\circ}$ to visualize the view in front of the user. This solution also reduces the computational complexity by decreasing the necessary orthomaps from one per wall to one from the ceiling. However, this approach does not allow the user to view the ceiling. If the view of the ceiling is also important, we can render additional orthomaps for the walls and track the ceiling using the planar walls. In this case, the performance gain mentioned earlier is no longer available.

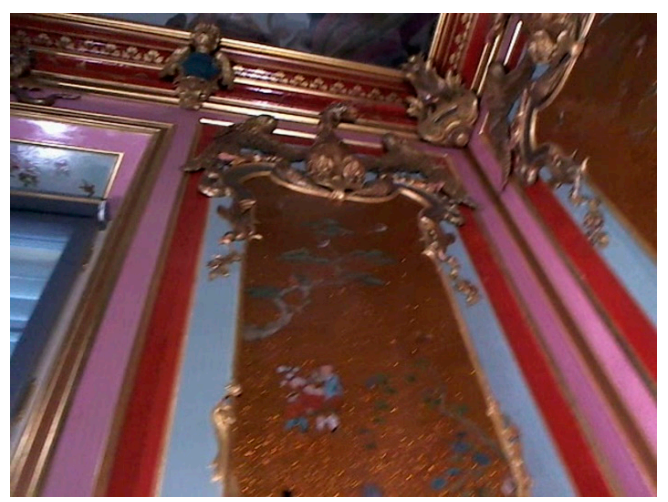

Figure 11. Example of the details under a high ceiling.

\section{Results}

To evaluate the accuracy of the proposed approach, we tested it on two printed posters: on a real-size fragment of a scanned Chinese Cabinet (Figure 12a) and actual size of the Al Fresco Cabinet (Figure 13a). Both posters are surrounded by ARUCO [36] fiducial markers. We recorded six different sequences that simulated the user behavior in front of the posters. In each frame, we estimated the camera pose twice, i.e., using keypoints from the poster and the corners of the extracted ARUCO markers. Both camera poses were then compared to evaluate the accuracy of our method. We considered the camera pose estimated from the ARUCO markers as our ground truth. Figures $12 b-d$ and $13 b-d$ show the estimated camera trajectories in each sequence. The quantitative results are listed in Tables 2 and 3. To measure the translation error, we calculated the Euclidean distance between the estimated camera poses from both methods and divided it by the L2 norm of the camera position estimated by ARUCO (Equation (5)).

$$
e_{t}=\frac{\sqrt{\left(x_{1}-x_{2}\right)^{2}+\left(y_{1}-y_{2}\right)^{2}+\left(z_{1}-z_{2}\right)^{2}}}{\sqrt{x_{1}^{2}+y_{1}^{2}+z_{1}^{2}}} \cdot 100 \%
$$

where:

$e_{t}$-translation error

$x_{1}, y_{1}, z_{1}$-coordinates of the ground-truth camera position

$x_{2}, y_{2}, z_{2}$-coordinates of the computed camera position 
We decided to normalize the translation error using the Euclidean distance from the marker to the camera because if the ratio between the error and distance remains the same, the camera image would also stay the same, regardless of the distance to the marker. To estimate the rotation error, we first converted the rotation matrices into quaternions and then computed the angle difference between them. We considered the angle obtained from ARUCO as the ground truth. Let us define two quaternions $\left(q_{1}\right.$ and $\left.q_{2}\right)$ to represent the camera rotations from both methods.

$$
q_{1}=\left(x_{1}, x_{2}, x_{3}, x_{4}\right), q_{2}=\left(y_{1}, y_{2}, y_{3}, y_{4}\right)
$$

Then, let us compute:

$$
q_{3}\left(z_{1}, z_{2}, z_{3}, z_{4}\right)=q_{1} \cdot \operatorname{conj}\left(q_{2}\right) .
$$

Finally, let us compute the rotation error in radians as:

$$
e_{r}=2 \cdot \operatorname{acos}\left(z_{1}\right)
$$

To examine the quality of the pose-estimation algorithm used in CATCHA, we decided to use statistical analysis. In each sequence, we calculated the four quartiles of the errors (Q1-Q4) and computed the interquartile range $(I Q R)$ of our errors to determine how many pose estimations we could consider as gross errors.

$$
I Q R=Q 3-Q 1
$$

To determine $I Q R$, we computed both the upper (UF) and lower (LF) fences with $k=1.5$ and marked the pose estimation errors that exceeded the upper limit as gross errors.

$$
\begin{aligned}
& U F=Q 3+k \cdot I Q R \\
& L F=Q 1-k \cdot I Q R
\end{aligned}
$$

The mean error was below $2.5 \%$ in the translation, and it was below $1.5^{\circ}$ in the rotation. The error values in each sequence are shown in Figure 14. In each case, the level of outliers was approximately $5 \%$ for the King's Chinese Cabinet and approximately $1 \%$ for the Al Fresco Cabinet. We note that the

\begin{tabular}{|c|c|c|c|c|c|c|}
\hline Sequence & 1 & & 2 & & 3 & \\
\hline \multirow[t]{2}{*}{ Number of Frames } & 182 & & 620 & & 483 & \\
\hline & Translation [\%] & Rotation $\left[{ }^{\circ}\right]$ & Translation [\%] & Rotation $\left[{ }^{\circ}\right]$ & Translation [\%] & Rotation $\left[{ }^{\circ}\right]$ \\
\hline Median & 0.71 & 0.45 & 0.95 & 0.61 & 0.99 & 0.69 \\
\hline Average & 0.83 & 0.54 & 1.19 & 0.80 & 1.26 & 0.90 \\
\hline Min & 0.14 & 0.03 & 0.05 & 0.03 & 0.08 & 0.06 \\
\hline Max & 3.50 & 2.20 & 9.03 & 6.54 & 6.93 & 5.43 \\
\hline Q1 & 0.44 & 0.28 & 0.57 & 0.35 & 0.62 & 0.41 \\
\hline Q3 & 1.02 & 0.69 & 1.50 & 1.00 & 1.55 & 1.08 \\
\hline IQR & 0.58 & 0.41 & 0.94 & 0.65 & 0.93 & 0.67 \\
\hline Lower fence $(k=1.5)$ & -0.44 & -0.34 & -0.84 & -0.62 & -0.78 & -0.59 \\
\hline Upper fence $(k=1.5)$ & 1.90 & 1.31 & 2.90 & 1.97 & 2.94 & 2.08 \\
\hline Outliers & 8 & 8 & 26 & 28 & 23 & 30 \\
\hline$\%$ of outliers & 4.4 & 4.4 & 4.2 & 4.5 & 4.8 & 6.2 \\
\hline
\end{tabular}
erroneous single-pose estimations were rejected and filtered using the visualization module so as not to affect the quality of the user experience. In our test procedure, we skipped the frames where only the markers in one row or one column were visible to avoid a situation where the reference pose was computed with a high rotation error.

Table 2. Statistical accuracy evaluation of the three analyzed sequences of the King's Chinese Cabinet. 


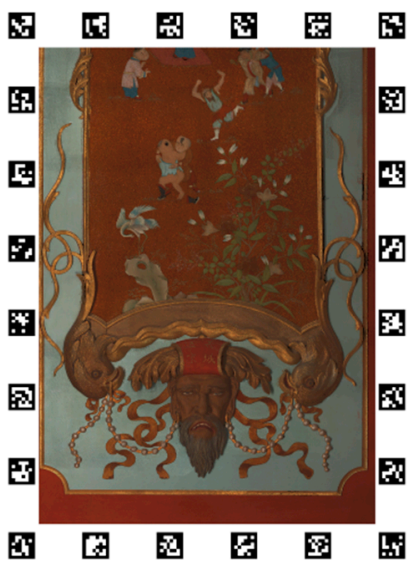

(a)

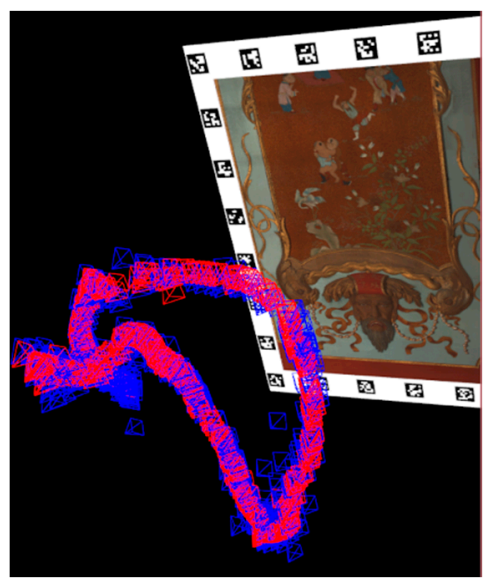

(c)

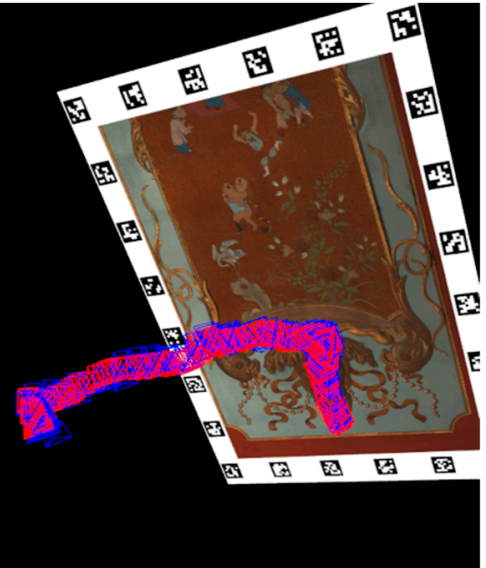

(b)

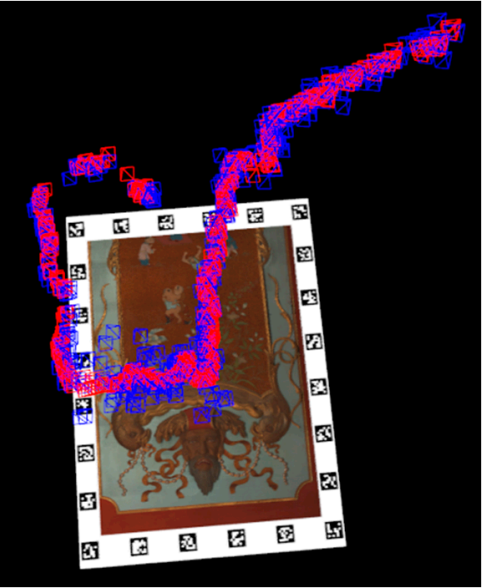

(d)

Figure 12. Algorithm evaluation of the King's Chinese Cabinet. (a) Poster used to test the accuracy. (b-d) Camera poses in each sequence (red-ground truth; blue-our method).

Table 3. Statistical accuracy evaluation of the three analyzed sequences of the Al Fresco Cabinet.

\begin{tabular}{|c|c|c|c|c|c|c|}
\hline Sequence & 4 & & 5 & & 6 & \\
\hline \multirow[t]{2}{*}{ Number of Frames } & 353 & & 391 & & 209 & \\
\hline & Translation [\%] & Rotation $\left[{ }^{\circ}\right]$ & Translation [\%] & Rotation $\left[{ }^{\circ}\right]$ & Translation [\%] & Rotation $\left[{ }^{\circ}\right]$ \\
\hline Median & 2.18 & 1.33 & 1.86 & 1.28 & 2.04 & 1.35 \\
\hline Average & 2.28 & 1.48 & 2.04 & 1.46 & 2.26 & 1.43 \\
\hline Min & 0.15 & 0.13 & 0.10 & 0.04 & 0.20 & 0.09 \\
\hline Max & 5.65 & 4.89 & 7.66 & 4.88 & 7.04 & 3.95 \\
\hline Q1 & 1.38 & 0.92 & 0.93 & 0.66 & 1.19 & 0.80 \\
\hline Q3 & 3.03 & 1.95 & 2.79 & 2.04 & 3.12 & 2.00 \\
\hline IQR & 1.65 & 1.04 & 1.86 & 1.38 & 1.92 & 1.20 \\
\hline Lower fence $(k=1.5)$ & -1.10 & -0.64 & -1.87 & -1.41 & -1.69 & -1.01 \\
\hline Upper fence $(k=1.5)$ & 5.51 & 3.51 & 5.59 & 4.11 & 6.00 & 3.81 \\
\hline Outliers & 1 & 6 & 4 & 2 & 2 & 1 \\
\hline$\%$ of outliers & 0.3 & 1.7 & 1.0 & 0.5 & 1.0 & 0.5 \\
\hline
\end{tabular}




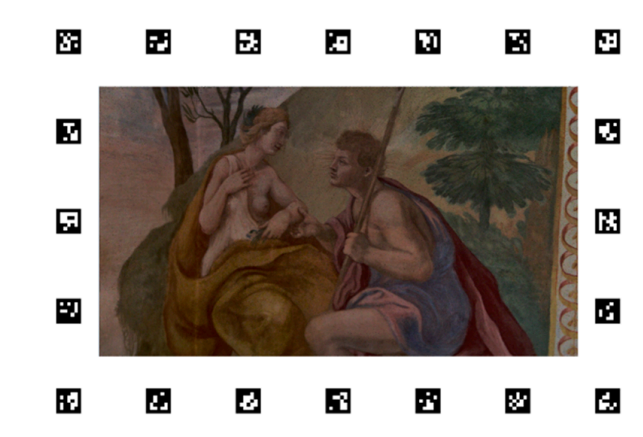

(a)

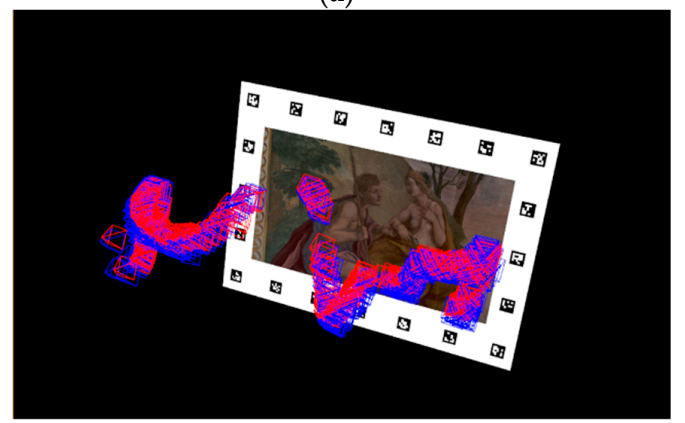

(c)

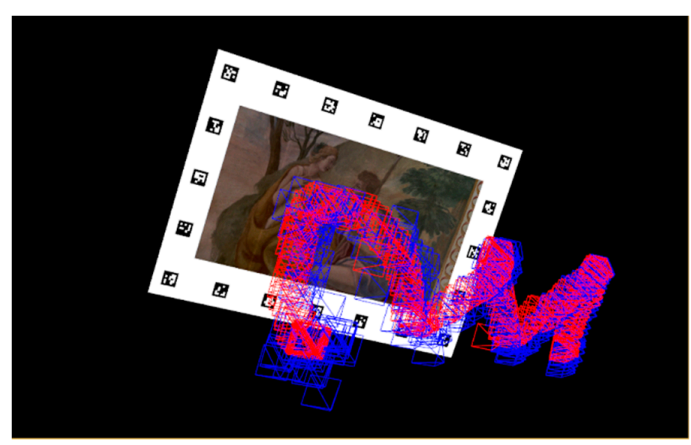

(b)

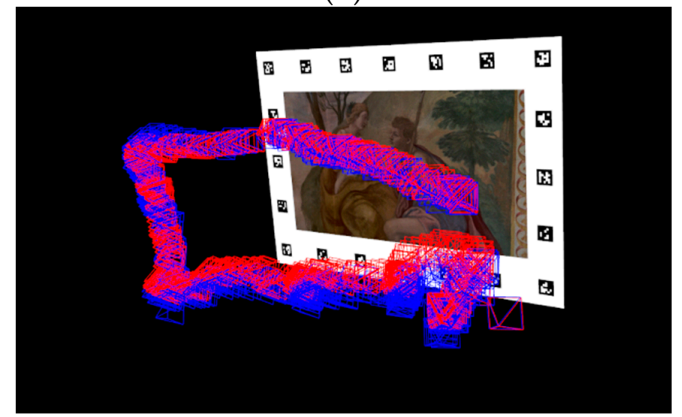

(d)

Figure 13. Algorithm evaluation of the Al Fresco Cabinet. (a) Poster used to test the accuracy. (b-d) Camera poses in each sequence (red-ground truth; blue-our method).

Translation error for Seq. 1

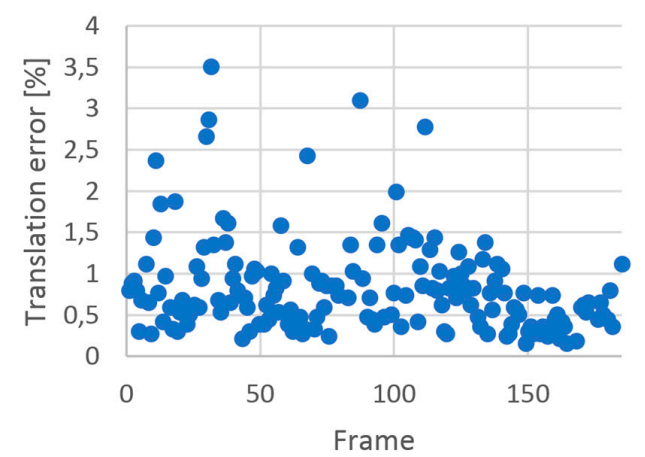

(a)

Translation error for Seq. 2

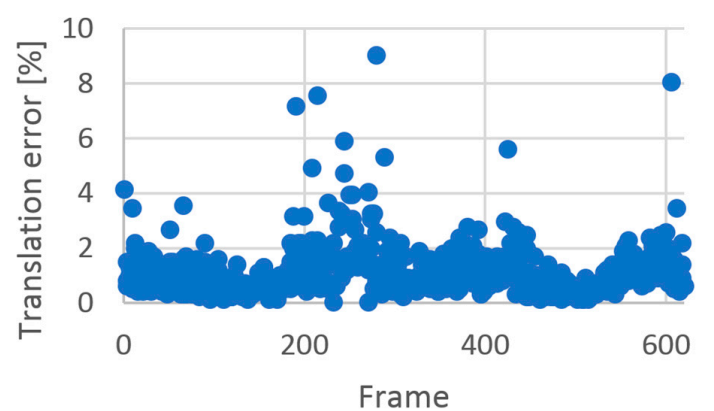

(c)
Rotation error for Seq. 1

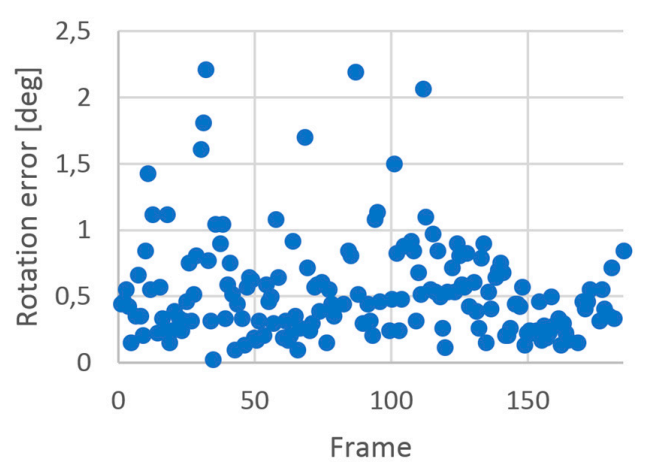

(b)

Rotation error for Seq. 2

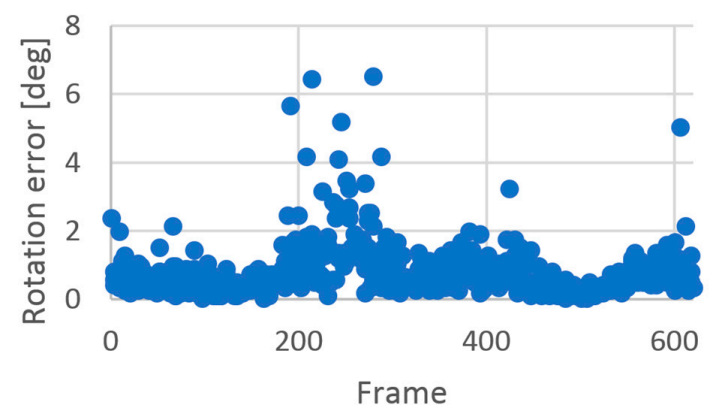

(d)

Figure 14. Cont. 
Translation error for Seq. 3

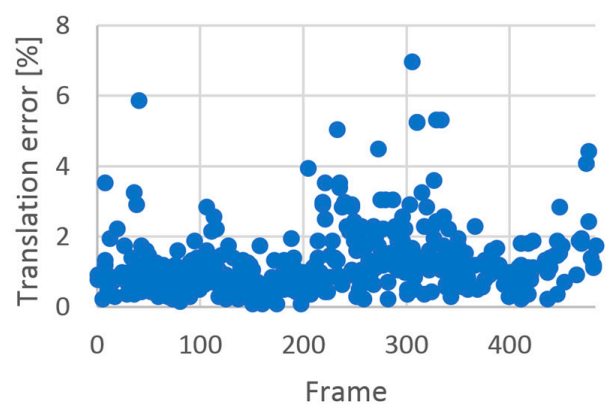

(e)

Translation error for Seq. 4

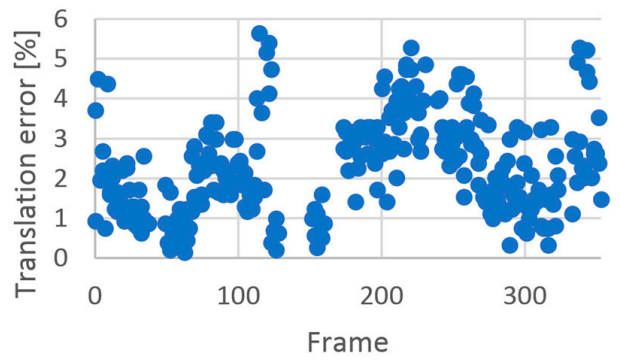

(g)

Translation error for Seq. 5

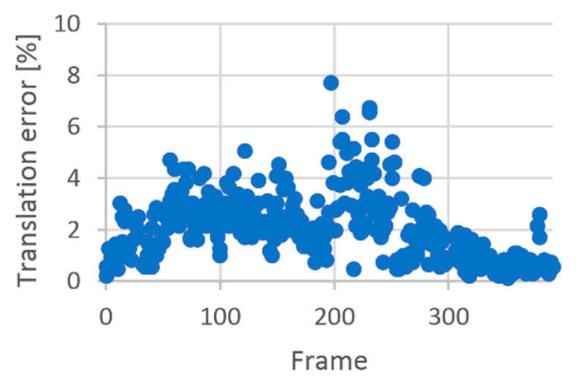

(i)

\section{Translation error for Seq. 6}

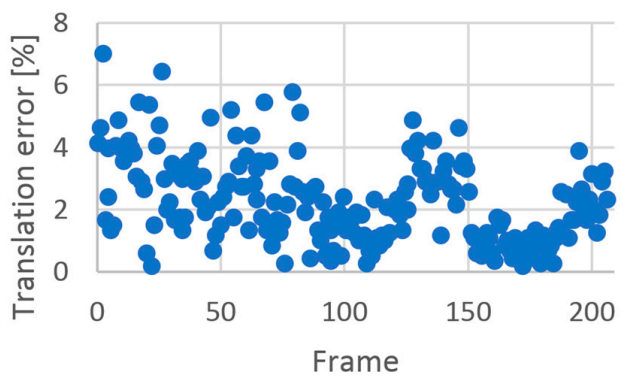

(k)
Rotation error for Seq. 3

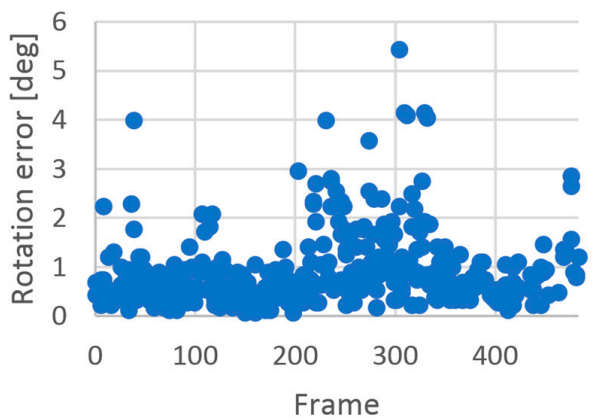

(f)

Rotation error for Seq. 4

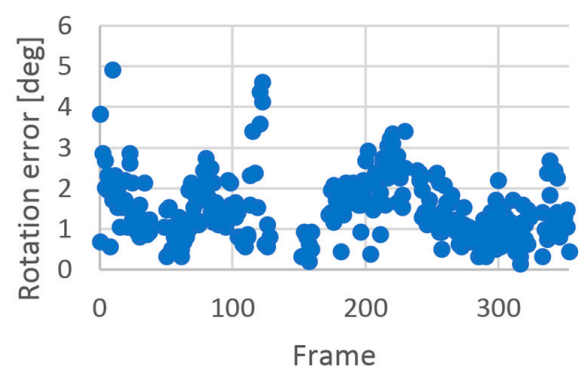

(h)

Rotation error for Seq. 5

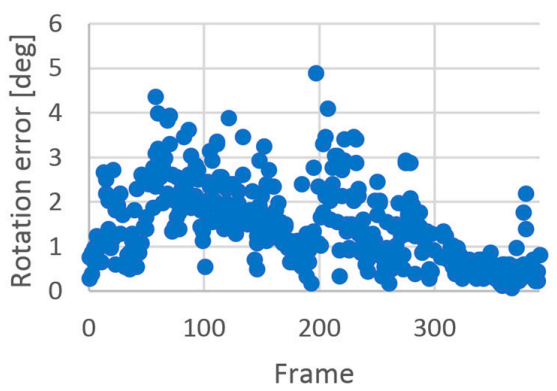

(j)

Rotation error for Seq. 6

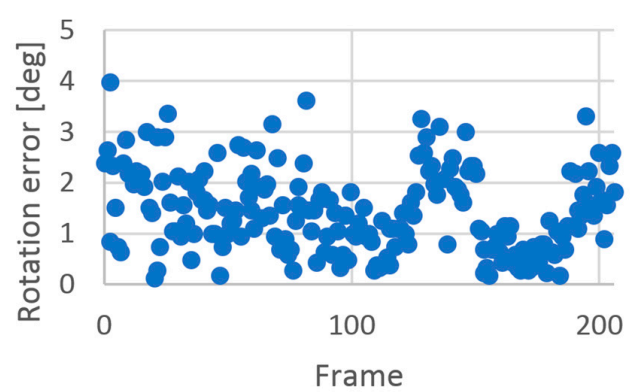

(1)

Figure 14. Error values in each test sequence. Left (a), (c), (e), (g), (i), and (k). Translation errors for sequences 1-6, respectively. Right $(\mathbf{b}),(\mathbf{d}),(\mathbf{f}),(\mathbf{h}),(\mathbf{j})$, and (1). Rotation errors for sequences $1-6$, respectively.

With respect to the application performance in the museum, by implementing the setup mentioned in Section 3, we achieved a mean frame rate of over $15 \mathrm{~Hz}$ directly using the SIFT descriptors on a mobile device. The mean frame rate increases up to $40 \mathrm{~Hz}$ using remote processing. This value is 
sufficient for smooth AR experience because many cameras on commercial devices and webcams have a maximum frame rate of up to $30 \mathrm{~Hz}$.

\section{Discussion}

Cultural heritage objects are more or less under strict protection, and their limitations must be taken into consideration while designing AR solutions. The King's Chinese Cabinet is an example of an interior under a very strict protection because of the very fragile pigments on the walls. The paintings may be damaged if illuminated by an infrared or ultraviolet light. In addition, the maximum permissible level of visible light is limited and the room is protected from any illumination sources, including sunlight. The presented restrictions forbid the use of RGBD cameras that emit infrared light. In contrast to the solutions in which light can be fully adjusted, such as those in shopping galleries, we are forced to limit the amount of light. To overcome the problem of darkness in the Cabinet, we could increase the exposure time of the camera or increase the gain. The former results in motion blur, and the latter increases noise. We decided to adjust the gain of the camera, but this also decreased the quality of the extracted and matched keypoints. We believe that this decision may have influenced the results.

Compared with most of the state-of-the-art approaches that depend on the SfM or DP models and images used to build them, CATCHA can be used on any textured point cloud without prior knowledge of its origin or the data used to construct it. In addition, the developed method can be used on point clouds built using any method, including SfM or DP. This factor indicates that CATCHA is substantially more versatile than many existing approaches. CATCHA is obviously limited to interiors with rich matte textures on the walls because its camera pose estimation algorithm relies on the keypoints extracted from the images. Line-based approach may be used if no textures are available or the surfaces are very glossy. CATCHA builds model from orthomaps that can be rendered from any model, regardless of its registration method and works well with interiors with perpendicular walls and ceilings. With regard to more sophisticated interior geometry (e.g., dome viewed from the inside), a much higher number of orthomaps may be required to cover the entire geometry and possible viewing angles. In this case, the model size and matching time increases. For larger models, more sophisticated matching strategies should be adopted. For those cases, the $k$-means-based descriptor matching, proposed by Nister and Stewenius [29], or matching using only a part of the original model based on the previous frames with a region-growing may be used.

Because CATCHA relies on a scanned 3D model of the tracked interior, if a significant change occurs in the interior's appearance between the current state and the registered state (e.g. additional furniture), the 3D model has to be updated. Minor changes can be handled effectively, either in the matches-filtration step or by the RANSAC camera pose estimation algorithm. In the first case, unknown points should fail the ratio test due to a high distance to known points from the orthomap, and the ratio between the first and second nearest point would be close to 1 . One of the possible solutions would be to integrate a SLAM-based tracking module to detect potential significant changes in model geometry or to track the model in areas that are poorly covered on the renders.

We partially overcome the problem of high-viewing angles by dividing the interiors into two groups, as mentioned in the previous section. However, this solution does not ensure that a camera pose would be established, regardless of the viewing angle. The first approach is limited by the viewing angle (Figure 11), and the second approach is limited by the fact that the camera has to point in the direction of the ceiling. In the Cabinet example, where the ceiling is approximately $6 \mathrm{~m}$ high, we examined both strategies mentioned in Section 3. The second strategy resulted in better user experience because the ceiling was always nearly parallel to the camera sensor plane. Additionally, keypoints were extracted and more robustly matched. Further, in the case of this particular interior, the walls contain much gilding that caused reflections in the camera frame, which, in combination with high viewing angles, resulted in less stable keypoints. 
Because CATCHA uses RANSAC to eliminate outliers in the matches between the model and current camera frame, its performance depends on the number of points after filtration of the camera image. If this number is extraordinarily high, the frame rate may significantly drop. To eliminate this problem, different outlier rejection strategies such as that in [37] may be applied. The problem of a high outlier ratio can be partially handled by higher orthomap quality because the lesser the noise is, the more accurate is the keypoint matching and thus the fewer are the outliers. The experimental results presented in the previous section showed that the shapes of the translation and rotation errors (Figure 14) were almost identical because translation and rotation were simultaneously estimated and one compensated the other. The mean and median error values obtained in the King's Chinese Cabinet were much lower than those obtained in the Al Fresco Cabinet because the quality of the registered texture of the latter model was lower. Despite this condition, the outlier ratios were higher in the King's Chinese Cabinet because much gilding and many other shiny surfaces were present that could cause unstable keypoints. The reason for the high error values on certain frames was that the matched keypoints populated only part of the image (Figure 15). Because of the non-uniform distribution of points used as an input to the $\mathrm{PnP}$ algorithm, the solution may fall in the local minimum, instead of the global minimum. The error distribution does not depend on a specific camera layout but rather on the configuration of matched keypoints found in the image. Single-error camera-pose estimations did not affect the animation quality because the camera path was smoothed using Gaussian filtration. In both analyzed interiors, the outlier ratios and error values were sufficiently low to allow smooth and precise visualization of the details.

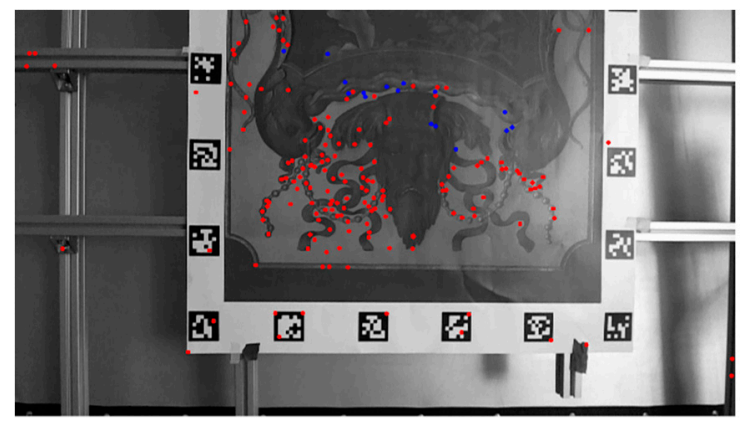

(a)

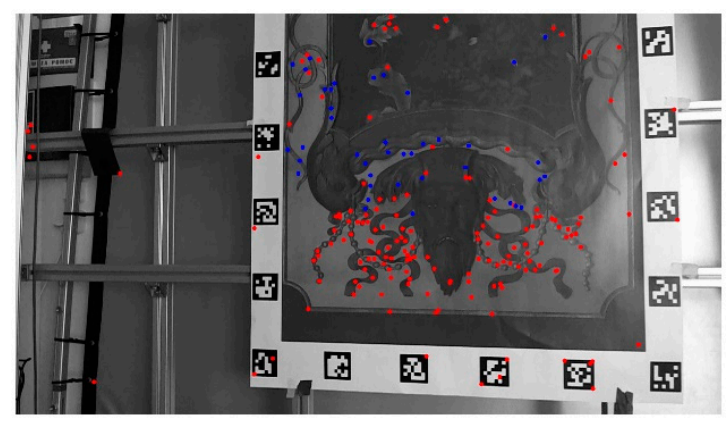

(b)

Figure 15. Point distribution: blue-inliers; red-outliers. (a) Non-uniform inliers are located only on a part of an image. (b) Uniform point distribution.

Beside the developed AR application allows the user to visually compare two states of the interior, the presented algorithm may be a base of many potential AR applications for professionals such as interactive model analysis (e.g. real-time curvature visualization and multispectral data mapping), presentations of interior design or interactive annotations for cultural heritage restorers. Ordinary visitors may be interested in simple augmentation of the interior with digital content, such as virtual guides who could walk around the interior and tell the story of the location. That would make the sightseeing more immersive.

\section{Conclusions}

We propose CATCHA - a novel AR camera tracking method inside cultural heritage interiors that satisfies very strict preservation requirements. The main novelty of this method is its orthographicbased data preparation that reduces the model complexity and does not depend on a point cloud registration method. We also propose a method that estimates the optimal orthographic resolution, which depends on real-world interior dimensions, camera FOV, and predicted optimal viewing distance. CATCHA was tested in two cultural heritage interiors in the Museum of King Jan III's 
Palace at Wilanów, Warsaw, namely, the King's Chinese Cabinet and Al Fresco Cabinet. In our test, we achieved a mean error of below $2.5 \%$ in translation and below $1.5^{\circ}$ in rotation. The accuracy of the camera-pose estimation algorithm is sufficient to provide visual feedback to the user. In our future work, we plan to examine different matching and data preparation strategies to improve robustness. One of the possible extensions may be to simultaneously track lines and keypoints. This approach may be helpful in handling glossy or textureless surfaces. Another direction of development can be automation of the process of mask generation using, for instance, machine learning methods. Finally, we plan to test CATCHA in different interiors and reduce the computational complexity to achieve a $60 \mathrm{~Hz}$ frame rate.

Author Contributions: Funding acquisition, R.S.; Investigation, P.S. and J.M.; Resources, E.B.; Software, P.S.; Supervision, R.S.; Validation, P.S. and E.B.; Visualization, J.M.; Writing—original draft, P.S.; Writing—review \& editing, R.S.

Funding: This work was partially supported by the Ministry of Culture and National Heritage (Poland), by the KULTURA+ framework, and by the project "Revitalization and digitalization Wilanów, the only Baroque royal residence in Poland," (grant No. POIS.11.01.00-00.068/14) which is co-financed by the European Union within the Infrastructure and Environment Operational Programme and Statutory Work of the Warsaw University of Technology.

Acknowledgments: We would like to thank Marta Nowak for providing the implementation of network transmission used for remote tracking.

Conflicts of Interest: The authors declare no conflict of interest.

\section{References}

1. Bekele, M.K.; Pierdicca, R.; Frontoni, E.; Malinverni, E.S.; Gain, J. A Survey of Augmented, Virtual, and Mixed Reality for Cultural Heritage. J. Comput. Cult. Herit. 2018, 11, 1-36. [CrossRef]

2. Sitnik, R.; Bunsch, E.; Mączkowski, G.; Załuski, W. Towards automated, high resolution 3D scanning of large surfaces for cultural heritage documentation. In ISET/SPIE Symposium on Electronic Imaging: Science ETechnology, ISET; Society for Imaging Science and Technology: Springfield, VA, USA, 2016.

3. Costantino, D.; Angelini, M.G.; Caprino, G. Laser Scanner Survey of An Archaeological Site: Scala Di Furno (Lecce, Italy). Int. Arch. Photogramm. Remote Sens. Spat. Inf. Sci. 2010, 38, 178-183.

4. Aicardi, I.; Chiabrando, F.; Maria Lingua, A.; Noardo, F. Recent trends in cultural heritage 3D survey: The photogrammetric computer vision approach. J. Cult. Herit. 2018, 32, 257-266. [CrossRef]

5. Marchand, E.; Uchiyama, H.; Spindler, F. Pose estimation for augmented reality: A hands-on survey. IEEE Trans. Vis. Comput. Graph. 2016, 22, 2633-2651. [CrossRef] [PubMed]

6. Pauwels, K.; Rubio, L.; Diaz, J.; Ros, E. Real-time model-based rigid object pose estimation and tracking combining dense and sparse visual cues. In Proceedings of the IEEE Computer Society Conference on Computer Vision and Pattern Recognition, Portland, OR, USA, $23-28$ June 2013; pp. 2347-2354.

7. Fuentes-Pacheco, J.; Ruiz-Ascencio, J.; Rendón-Mancha, J.M. Visual simultaneous localization and mapping: A survey. Artif. Intell. Rev. 2012, 43, 55-81. [CrossRef]

8. Zeisl, B.; Sattler, T.; Pollefeys, M. Camera Pose Voting for Large-Scale Image-Based Localization. In Proceedings of the 2015 IEEE International Conference on Computer Vision (ICCV), Santiago, Chile, 7-13 December 2015; pp. 2704-2712.

9. Rubio, A.; Villamizar, M.; Ferraz, L.; Ramisa, A.; Sanfeliu, A. Efficient Monocular Pose Estimation for Complex 3D Models. In Proceedings of the 2015 IEEE International Conference on Robotics and Automation (ICRA), Seattle, WA, USA, 26-30 May 2015; Volume 2, pp. 1397-1402.

10. Yang, G.; Becker, J.; Stewart, C.V. Estimating the location of a camera with respect to a 3D model. In Proceedings of the 3DIM 2007 6th International Conference on 3-D Digital Imaging and Modeling, Montreal, QC, Canada, 21-23 August 2007; pp. 159-166.

11. Guan, W.; You, S.; Pang, G. Estimation of camera pose with respect to terrestrial LiDAR data. In Proceedings of the IEEE Workshop on Applications of Computer Vision, Tampa, FL, USA, 15-17 January 2013; pp. 391-398.

12. Wu, C.; Fraundorfer, F.; Frahm, J.-M.; Pollefeys, M. 3D model search and pose estimation from single images using VIP features. In Proceedings of the 2008 IEEE Computer Society Conference on Computer Vision and Pattern Recognition Workshops, Anchorage, AK, USA, 23-28 June 2008; pp. 1-8. [CrossRef] 
13. Lowe, D.G. Distinctive image features from scale-invariant keypoints. Int. J. Comput. Vis. 2004, 60, 91-110. [CrossRef]

14. Taketomi, T.; Sato, T.; Yokoya, N. Real-time and accurate extrinsic camera parameter estimation using feature landmark database for augmented reality. Comput. Graph. 2011, 35, 768-777. [CrossRef]

15. Jaramillo, C.; Dryanovski, I.; Valenti, R.G.; Xiao, J. 6-DoF pose localization in 3D point-cloud dense maps using a monocular camera. In Proceedings of the 2013 IEEE International Conference on Robotics and Biomimetics, ROBIO 2013, Shenzhen, China, 12-14 December 2013; pp. 1747-1752.

16. Crombez, N.; Caron, G.; Mouaddib, E.M. Using dense point clouds as environment model for visual localization of mobile robot. In Proceedings of the 2015 12th International Conference on Ubiquitous Robots and Ambient Intelligence (URAI), Goyang, Korea, 28-30 October 2015; pp. 40-45. [CrossRef]

17. Rambach, J.; Pagani, A.; Schneider, M.; Artemenko, O.; Stricker, D. 6DoF Object Tracking based on 3D Scans for Augmented Reality Remote Live Support. Computers 2018, 7, 6. [CrossRef]

18. Vanoni, D.; Seracini, M.; Kuester, F. ARtifact: Tablet-Based Augmented Reality for Interactive Analysis of Cultural Artifacts. In Proceedings of the 2012 IEEE International Symposium on Multimedia, Irvine, CA, USA, 10-12 December 2012; pp. 44-49.

19. PTC Inc. Vuforia Library. Available online: https://www.vuforia.com/ (accessed on 3 October 2018).

20. Gîrbacia, F.; Butnariu, S.; Orman, A.P.; Postelnicu, C.C. Virtual restoration of deteriorated religious heritage objects using augmented reality technologies. Eur. J. Sci. Theol. 2013, 9, 223-231.

21. The Fraunhofer Institute for Computer Graphics Research IGD Instant Player. Available online: http:/ / www. instantreality.org/ (accessed on 3 October 2018).

22. Verykokou, S.; Ioannidis, C.; Kontogianni, G. 3D visualization via augmented reality: The case of the middle stoa in the ancient agora of Athens. In Lecture Notes in Computer Science, Proceedings of the Euro-Mediterranean Conference, Limassol, Cyprus, 3-8 November 2014; Springer: Berlin, Germany, 2014; Volume 8740, pp. 279-289101007978.

23. Battini, C.; Landi, G. 3D tracking based augmented reality for cultural heritage data management. Int. Arch. Photogramm. Remote Sens. Spat. Inf. Sci. 2015, 40, 375. [CrossRef]

24. Inglobe Technologies ARmedia 3D SDK. Available online: http://dev.inglobetechnologies.com/index.php (accessed on 3 October 2018).

25. Lech, K.; Maczkowski, G.; Bunsch, E. Color Correction in 3D Digital Documentation: Case Study. In Image and Signal Processing; Mansouri, A., Nouboud, F., Chalifour, A., Mammass, D., Meunier, J., Elmoataz, A., Eds.; Springer International Publishing: Cham, Switzerland, 2016; pp. 388-397.

26. CharLS. CharLS Library. Available online: https://github.com/team-charls/charls (accessed on 3 October 2018).

27. Rublee, E.; Rabaud, V.; Konolige, K.; Bradski, G. ORB: An efficient alternative to SIFT or SURF. In Proceedings of the IEEE International Conference on Computer Vision, Barcelona, Spain, 6-13 November 2011; pp. 2564-2571.

28. Sibbing, D.; Sattler, T.; Leibe, B.; Kobbelt, L. SIFT-realistic rendering. In Proceedings of the 2013 International Conference on 3D Vision, 3DV 2013, Seattle, WA, USA, 29 June-1 July 2013; pp. 56-63.

29. Nister, D.; Stewenius, H. Scalable recognition with a vocabulary tree. In Proceedings of the IEEE Computer Society Conference on Computer Vision and Pattern Recognition, New York, NY, USA, 17-22 June 2006; Volume 2, pp. 2161-2168.

30. Lepetit, V.; Moreno-Noguer, F.; Fua, P. EPnP: An Accurate O(n) Solution to the PnP Problem. Int. J. Comput. Vis. 2008, 81, 155. [CrossRef]

31. Gao, X.S.; Hou, X.R.; Tang, J.; Cheng, H.F. Complete solution classification for the perspective-three-point problem. IEEE Trans. Pattern Anal. Mach. Intell. 2003, 25, 930-943. [CrossRef]

32. Siekański, P.; Bunsch, E.; Sitnik, R. Seeing the past: An augmented reality application for visualization the previous state of cultural heritage locations. In Electronic Imaging; Society for Imaging Science and Technology: Springfield, VA, USA, 2018; Volume 2018, pp. 4521-4524.

33. Bay, H.; Tuytelaars, T.; Van Gool, L. SURF: Speeded Up Robust Features. In Lecture Notes in Computer Science, European Conference on Computer Vision, Graz, Austria, 7-13 May 2006; Springer: Berlin, Germany, 2006; pp. 404-417. 
34. Irschara, A.; Zach, C.; Frahm, J.M.; Bischof, H. From structure-from-motion point clouds to fast location recognition. In Proceedings of the 2009 IEEE Computer Society Conference on Computer Vision and Pattern Recognition Workshops, CVPR Workshops 2009, Miami, FL, USA, 20-25 June 2009; pp. 2599-2606.

35. Morel, J.-M.; Yu, G. ASIFT: A New Framework for Fully Affine Invariant Image Comparison. SIAM J. Imaging Sci. 2009, 2, 438-469. [CrossRef]

36. Garrido-Jurado, S.; Muñoz-Salinas, R.; Madrid-Cuevas, F.J.; Marín-Jiménez, M.J. Automatic generation and detection of highly reliable fiducial markers under occlusion. Pattern Recognit. 2014, 47, 2280-2292. [CrossRef]

37. Ferraz, L.; Binefa, X.; Moreno-Noguer, F. Very fast solution to the PnP problem with algebraic outlier rejection. In Proceedings of the IEEE Computer Society Conference on Computer Vision and Pattern Recognition, Columbus, OH, USA, 23-28 June 2014; pp. 501-508.

2018 by the authors. Licensee MDPI, Basel, Switzerland. This article is an open access article distributed under the terms and conditions of the Creative Commons Attribution (CC BY) license (http:/ / creativecommons.org/licenses/by/4.0/). 